\title{
Molecular dyes used for surgical specimen margin orientation allow for intraoperative optical assessment during breast conserving surgery
}

David M. McClatchy, III

Venkataramanan Krishnaswamy

Stephen C. Kanick

Jonathan T. Elliott

Wendy A. Wells

Richard J. Barth, Jr.

Keith D. Paulsen

Brian W. Pogue 


\section{Molecular dyes used for surgical specimen margin orientation allow for intraoperative optical assessment during breast conserving surgery}

\author{
David M. McClatchy III, ${ }^{\mathrm{a}, \star}$ Venkataramanan \\ Krishnaswamy, ${ }^{\text {a }}$ Stephen C. Kanick, ${ }^{a}$ \\ Jonathan T. Elliott, ${ }^{a}$ Wendy A. Wells, ${ }^{b}$

 \\ Brian W. Pogue a,c \\ aDartmouth College, Thayer School of Engineering, 14 Engineering \\ Drive, Hanover, New Hampshire 03755, United States \\ bDartmouth College, Department of Pathology, Geisel School of \\ Medicine, 1 Rope Ferry Road, Hanover, New Hampshire 03755, \\ United States \\ 'Dartmouth College, Department of Surgery, Geisel School of \\ Medicine, 1 Rope Ferry Road, Hanover, New Hampshire 03755, \\ United States \\ dDartmouth College, Department of Diagnostic Radiology, \\ Geisel School of Medicine, 1 Rope Ferry Road, Hanover, \\ New Hampshire 03755, United States
}

Abstract. A variety of optical techniques utilizing nearinfrared (NIR) light are being proposed for intraoperative breast tumor margin assessment. However, immediately following a lumpectomy excision, the margins are inked, which preserves the orientation of the specimen but prevents optical interrogation of the tissue margins. Here, a workflow is proposed that allows for both NIR optical assessment following full specimen marking using molecular dyes which have negligible absorption and scattering in the NIR. The effect of standard surgical inks in contrast to molecular dyes for an NIR signal is shown. Further, the proposed workflow is demonstrated with full specimen intraoperative imaging on all margins directly after the lumpectomy has been excised and completely marked. This work is an important step in the path to clinical feasibility of intraoperative breast tumor margin assessment using NIR optical methods without having to compromise on the current clinical practice of inking resected specimens for margin orientation. (c) The Authors. Published by SPIE under a Creative Commons Attribution 3.0 Unported License. Distribution or reproduction of this work in whole or in part requires full attribution of the original publication, including its DOI. [DOI: 10.1117/1.JBO.20.4.040504]

Keywords: breast tumor margins; breast conserving surgery; tissue diagnostics; near-infrared; molecular dyes.

Paper 140489LR received Jul. 29, 2014; accepted for publication Mar. 31, 2015; published online Apr. 22, 2015.

\footnotetext{
*Address all correspondence to: David M. McClatchy III, E-mail: david.m
} .mcclatchy.iii.th@dartmouth.edu
With advances in breast cancer screening technologies and the recent widespread adoption of neoadjuvant chemotherapy, an increasing number of patients are undergoing breast conserving surgery (BCS) in lieu of a full mastectomy. It has been shown that the long-term survival of patients who undergo BCS is equivalent to those who undergo a full mastectomy if all of the tumor margins are negative. ${ }^{1}$ This process is encouraging in that less invasive surgeries are completed as compared to full mastectomy, yet this smaller excision volume also presents added pressure on the clinical need to accurately ensure that there was a complete excision with no remaining residual cancer in the body. If a positive margin is diagnosed from the resection specimen after the procedure, then a second re-excision surgery is performed to remove further tissue at added cost and patient morbidity. Due to the lack of effective intraoperative surgical guidance and the difficulty in tumor localization with decreasing tumor size, re-excision rates have been reported to be as high as 20 to $40 \%{ }^{2}$ However, re-excision rates vary greatly between institutions because of differing protocols for what constitutes a positive margin. The current standard of care for BCS involves a preoperative wire localization of the tumor core, guided by mammography or ultrasound, as shown in Fig. 1. This wire is a landmark to aid in discerning the central location of the tumor during the procedure and a rough guide to the boundary. After the tumor is excised, its margins are immediately inked by the surgeon in order to preserve its orientation in the event that a second re-excision is required. ${ }^{3}$ Histopathological analysis performed over the following one to two days determines if there is cancerous involvement at the margins. Currently, a myriad of optical techniques are being investigated to intraoperatively determine if there is cancerous involvement at the margins. ${ }^{4-8}$ However, severe signal degradation by the surgical inks prevents all of these proposed techniques from potential clinical translation, so the subject of this letter is to outline the use of organic dyes which serve the same function as surgical inks but then allow spectroscopic imaging of the underlying tissue through most of the near-infrared (NIR) spectrum.

Given the prevalence of BCS, there is a clinical need for intraoperative surgical specimen margin assessment to augment pathological analysis, and hopefully to curb the high rate of reexcision procedures on subsequent days following the initial surgery. Because cancerous processes locally alter tissue ultrastructure in both epithelial and stromal structure, its presence can be detected through changes in the optical spectrum. ${ }^{9,10}$ Many microscopic optical techniques have been examined for intraoperative margin assessment of localized spots, including frozen section phase microscopy, optical coherence tomography, confocal microscopy, fluorescence imaging, Raman spectroscopic microscopy, and polarization. ${ }^{4-6}$ Macroscopic imaging of the entire tissue field has had limited study, but both raster scanning and spatial frequency domain imaging systems have recently shown promise. ${ }^{7,8}$ These studies have focused on the NIR spectrum of light due to the diminished absorption attenuation from surface blood on the specimen, and thus far, there have been many promising results demonstrating a realistic potential for future clinical use.

However, many of these studies involve a workflow of either obtaining optical tissue measurements before inking or after specimens are loafed into slices during postsurgical processing. While these workflows are acceptable for research investigations, the standard practice of inking the specimen during excision to preserve its orientation must be incorporated into these emerging 


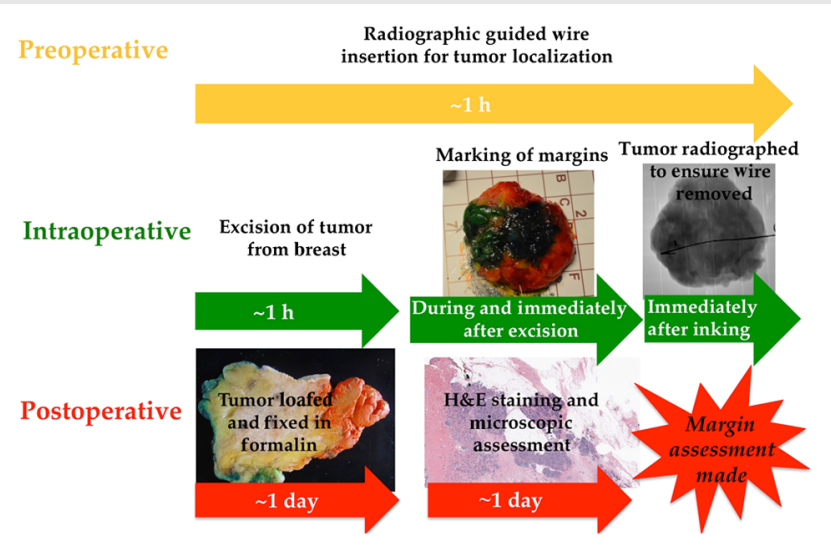

Fig. 1 A workflow diagram showing the typical phases of a breast conserving surgery. The arrows indicate the approximate time of each phase.

optical techniques in order for translation into the current standard of care for BCS. Marking the specimen margins during excision is critical as adipose tissue largely compromises the lumpectomy margin so they are extremely amorphous. In addition to their shapelessness, the presence of surface blood makes it very difficult to keep track of the margin orientation. If the orientation of the specimen was not preserved, then it would be unclear where to re-excise tissue in the event of a positive margin being found. Unfortunately, these surgical inks indefinitely limit optical probing of the tissue due to their extreme scattering and absorption properties (as seen in Fig. 2). Techniques to manually fix the specimen in place so that its orientation can be set without the use of ink have been reported, but these techniques are difficult as lumpectomies can drastically differ in both size and shape requiring many different fixing mechanisms. ${ }^{11}$ Furthermore, the margins of a given lumpectomy can be asymmetrical after removal from the anatomical support of the breast.

To more easily translate direct optical intraoperative margin assessment techniques into a standard BCS procedure with as little interference as possible, a marking system is needed that has negligible optical effects in the NIR wavelengths but still provides contrast under white light. Common surgical

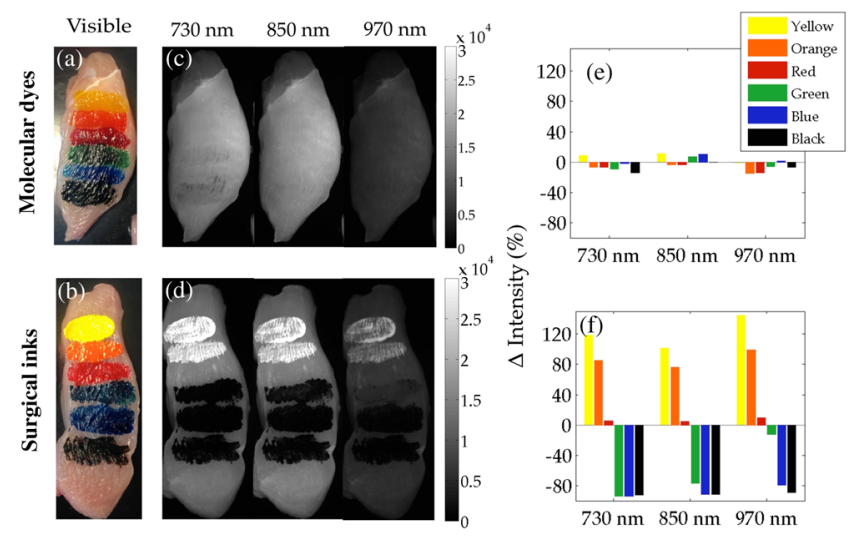

Fig. 2 White light color image of avian muscle phantom with six standard margin orientation colors of (a) molecular dyes and (b) surgical inks. Near-infrared wavelength $(730,850$, and $970 \mathrm{~nm})$ full-field imaging of the phantom with (c) molecular dyes and (d) surgical inks. Bar graph of the relative change in signal intensity for the (e) molecular dyes and (f) surgical inks between each color and surrounding unmarked tissue for each wavelength. inks are densely composed of particulate carbon and pigments leading to both strong absorption and scattering in the NIR. In this letter, molecular dyes are introduced which have a composition of a minimally scattering gel with molecular absorbers, which are Food, Drug \& Cosmetics food dyes that happen to only absorb in the visible spectrum. The difference in optical attenuation in the NIR between the molecular dyes and standard surgical inks can be seen in Fig. 2. Avian muscle tissue was marked with every color of a standard surgical ink (The Davidson System, Bradley Products, Inc., Bloomington, Minnesota) and a molecular dye (Ateco, Glen Cove, New York), which is simply a consumer food dye gel. The tissue was illuminated with three light-emitting diodes spanning the NIR (730, 850 , and $970 \mathrm{~nm}$ ) and imaged with a CCD which were part of a commercial planar imaging system (Modulated Imaging, Inc., Irvine, California). ${ }^{12}$ In Figs. 2(a) and 2(b), one can see under white illumination that both the surgical ink and molecular dyes have very similar visible contrast. But in Figs. 2(c) and 2(d), it is apparent that the surgical inks completely degrade the optical signal at all three wavelengths while the molecular dye appears virtually invisible except for faint absorption of the black dye at $730 \mathrm{~nm}$. Figures 2(e) and 2(f) show bar graphs for the relative change in signal intensity between each of the six markings and neighboring unmarked tissue for the NIR wavelength wide-field imaging. Figure 2(e) shows that the maximum change in signal intensity for the molecular dyes is roughly $15 \%$ for the black dye at $730 \mathrm{~nm}$. However, Fig. 2(f) shows that all of the surgical inks with the exception of red at all wavelengths and green at $970 \mathrm{~nm}$ have an absolute signal intensity change of at least $70 \%$ at all of the NIR wavelengths. The faint absorption of the black molecular dye is insignificant though as many of the potential optical techniques have a subdiffusive sensitivity where absorption effects are minimized. On the contrary, surgical inks are specifically manufactured so that they are visible under microscopic assessment. It should also be noted, however, that the watersoluble molecular dyes cannot survive standard tissue processing standards that precede histopathological assessment. As such, the molecular dyes simply act as a temporary proxy for the surgical inks to allow intraoperative optical assessment, after which the standard surgical inks are applied.

A pilot study was also conducted to introduce a potential clinical workflow that would allow for both intraoperative optical margin assessment and the surgeon to perform routine specimen orientation inking during resection, but with the molecular dyes. The major goals of this pilot study were to demonstrate that the proposed molecular dyes could be used as an effective temporary substitute to the traditional surgical inks in a clinical setting and, consequently, show that NIR optical probing could be performed in the presence of the molecular dyes after the margins were marked. The workflow for the study involved the surgeon marking the lumpectomy margins with the molecular dye during resection, and then all six of the margins were imaged in a room adjacent to the operating room (OR). After imaging, the specimen went back to the OR, where the surgeon applied standard surgical inks for standard histopathological assessment. All patients ( $n=6$ patients) gave informed consent to participate in this clinical trial, which was approved by the Dartmouth institutional review board. To demonstrate NIR optical probing through the molecular dyes, a previously described wide-field imaging technique called structured light scatteroscopy (SLS) was performed. ${ }^{13}$ This technique has been shown to have insensitivity to surface blood and enhanced 


\section{JBO Letters}

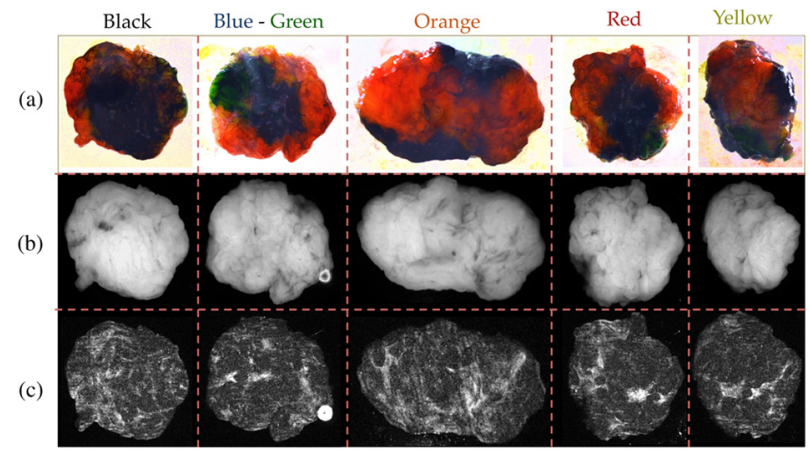

Fig. 3 Sample data set of full specimen intraoperative imaging through a glass slide after margins were completely marked with molecular dye. (a) Color photographs, (b) wide-field imaging with 850-nm light-emitting diodes, and (c) structured light scatteroscopy imaging $(850 \mathrm{~nm})$ with a sinusoidal pattern of $0.5 \mathrm{~mm}^{-1}$.

sensitivity to scattering, which was a significant diagnostic factor in previous breast tumor margin NIR imaging studies. ${ }^{7,8,13}$ However, retrospective analyses of SLS data and histopathology were not included as a part of this study, as the main goal was demonstrating the utility of molecular dyes for NIR methods aimed toward intraoperative breast tumor margin assessment. SLS involves illuminating high spatial frequency structured light and demodulating the perturbed pattern. ${ }^{13}$ For this study, a sinusoidal pattern with a spatial frequency of $0.5 \mathrm{~mm}^{-1}$ was illuminated, having an effective penetration depth of $\sim 0.3 \mathrm{~mm}$. The pattern was projected at three evenly spaced phase offsets of $0,2 \pi / 3,4 \pi / 3$, yielding images $I_{1}, I_{2}, I_{3}$, which were used to calculate the demodulated image $I_{d} \cdot{ }^{14}$

$I_{d}=\left(2^{1 / 2} / 3\right)\left[\left(I_{1}-I_{2}\right)^{2}+\left(I_{1}-I_{3}\right)^{2}+\left(I_{2}-I_{3}\right)^{2}\right]^{1 / 2}$.

In addition, standard full-field illumination imaging was performed by averaging $I_{1}, I_{2}$, and $I_{3}$. The acquisition time to image one face of the margin is only $\sim 1 \mathrm{~min}$, and the entire imaging procedure was $\sim 20 \mathrm{~min}$ with transporting and handling the tissue. Data were acquired with the same commercial NIR imaging system from Modulating Imaging, Inc., and the molecular dyes and surgical inks were also the same as used in the chicken breast phantom. Figure 3 shows the $850 \mathrm{~nm}$ channel collected for a single lumpectomy specimen. In Fig. 3(a), white light images show how the entire specimen is visible when marked with colored dye. The specimens were placed on a glass slide and imaged from below. Because of their shapelessness, often two previously orthogonal margins could be imaged at once, as is the case in Fig. 3 with the blue and green margins. Figures 3(b) and 3(c) show the diffuse full-field images and the demodulated images, $I_{d}$, respectively, for the $850 \mathrm{~nm}$ channel. While both sets of images in Figs. 3(b) and 3(c) show insensitivity to the molecular dyes, the SLS image in Fig. 3(c) shows high contrast features arising from local changes in light scattering from changes in tissue morphology. This presented workflow demonstrates feasibility for using molecular dyes as a temporary proxy for standard surgical inks with minimal interference to both NIR optical signals and the current standard of care BCS procedure.

Due to the increasing prevalence of BCS and accompanying high rates of re-excision, there is a strong clinical need for an intraoperative tumor margin assessment system. While many optical methods have been proposed and have demonstrated sensitivity in pilot studies, they fail to negotiate margin orientation inking. Using molecular dyes for full specimen marking after resection would allow for NIR optical assessment of the margins without significantly altering the current standard of care practice of BCS.

\section{References}

1. U. Veronesi et al., "Twenty-year follow-up of a randomized study comparing breast-conserving surgery with radical mastectomy for early breast cancer," N. Engl. J. Med. 347(16), 1227-1232 (2002).

2. R. G. Pleijhuis et al., "Obtaining adequate surgical margins in breastconserving therapy for patients with early-stage breast cancer: current modalities and future directions," Ann. Surg. Oncol. 16(10), 2717-2730 (2009).

3. G. R. Gibson et al., "A comparison of ink-directed and traditional whole-cavity re-excision for breast lumpectomy specimens with positive margins," Ann. Surg. Oncol. 8(9), 693-704 (2001).

4. J. Q. Brown et al., "Optical assessment of tumor resection margins in the breast," IEEE J. Sel. Topics Quantum Electron. 16(3), 530-544 (2010).

5. R. Patel et al., "Multimodal optical imaging for detecting breast cancer," J. Biomed. Opt. 17(6), 066008 (2012).

6. R. Patel et al., "Polarization sensitive multimodal imaging for detecting breast cancer," Cancer Res. 74, 4685-4693 (2014).

7. A. M. Laughney et al., "Scatter spectroscopic imaging distinguishes between breast pathologies in tissues relevant to surgical margin assessment," Clin. Cancer Res. 18(22), 6315-6325 (2012).

8. A. M. Laughney et al., "Spectral discrimination of breast pathologies in situ using spatial frequency domain imaging," Breast Cancer Res. 15(4), R61 (2013).

9. Y. L. Kim et al., "Low-coherent backscattering spectroscopy for tissue characterization," Appl. Opt. 44(3), 366-377 (2005).

10. V. Krishnaswamy et al., "Quantitative imaging of scattering changes associated with epithelial proliferation, necrosis, and fibrosis in tumors using microsampling reflectance spectroscopy," J. Biomed. Opt. 14(1), 014004 (2009).

11. T. Karni et al., "A device for real-time, intraoperative margin assessment in breast-conservation surgery," Am. J. Surg. 194, 467-473 (2007).

12. D. J. Cuccia et al., "Quantitation and mapping of tissue optical properties using modulated imaging," J. Biomed. Opt. 14(2), 024012 (2009).

13. V. Krishnaswamy et al., "Structured light scatteroscopy," J. Biomed. Opt. 19(7), 070504 (2014).

14. M. A. Neil, R. Juskaitis, and T. Wilson, "Method of obtaining optical sectioning by using structured light in a conventional microscope," Opt. Lett. 22(24), 1905-1907 (1997). 